\title{
La Carranga como escenario vivo de la tradición e identidad cultural local y regional del departamento de Boyacá
}

Francy Marizol Rojas Parra ${ }^{1}$

\begin{abstract}
Resumen
Este documento ${ }^{2}$ es el resultado de una investigación fundamentada en la memoria colectiva y la riqueza popular de fuentes simbólicas y representativas de la música carranguera como escenario principal de acontecimientos que aportan en la construcción de hechos y la formación de identidades, así como los diversos conflictos que convocan a la disgregación cultural, a través de la valoración del impacto histórico, social y cultural. También se busca establecer de qué manera influyen en las diferentes manifestaciones del patrimonio inmaterial, en los valores, creencias, usos, costumbres, expresiones y en la vida cotidiana de la población. Además de ello, pretende realizar un acercamiento a la autenticidad de los festejos, celebraciones populares y su influencia en la cotidianidad.
\end{abstract}

Palabras clave: riqueza popular, costumbres, música carranguera, música popular, expresiones Inmateriales, tradición.

1 Candidata a Magíster en Historia. UPTC. FRANCY MARIZOL ROJAS PARRA. Universidad Pedagógica Tecnológica De Colombia. Docente del área del Patrimonio Social y Cultural. Investigadora Cultural - Grupo Emprender U.P.T.C. Marisolrojas9@yahoo.es. Celular 3208368910 


\title{
The Carranga as a living stage of traditional, local and regional cultural identity of Bovaca Department (Boyaca, Colombia).
}

\begin{abstract}
This study aimed to evaluate the carragera music through historical, social and cultural impact assessment. This document is a product of a serious research based on collective memory and popular richness of symbolic and representative sources of carranguera music as main stage of events that bring in the facts construction and identity formation. In the same way in the different conflicts that attract cultural disaggregation. It was established the influence of these factors on different manifestations of intangible heritage, values, belief, custom, expressions and people's everyday life. Furthermore, this study approached the authenticity of festivities, popular celebrations and their influence on daily life.
\end{abstract}

Key words: popular richness, customs, Carranguera music, popular music, intangible expressions, tradition.

Recibido: 12 de febrero de 2013

Aceptado: 03 de diciembre de 2013

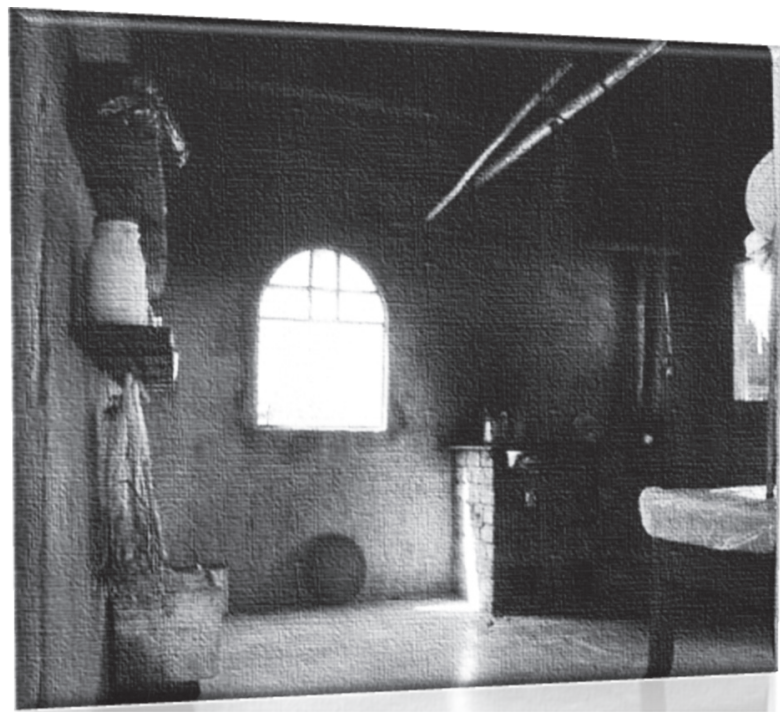

Foto: Interior de una Casa

Boyacense - Sotaquirá 


\section{Revista de}

investigaciones UNAD

Volumen 12. Número 2. Diciembre 2013

\section{LA MÚSICA: IDENTIDAD LOCAL Y REGIONAL}

Somos hablares, historias copla, canto y poseías, eso es lo que les traemos, eso es la carrangueria, sinfónicamente hablando, un canto hermoso a la vida.

Jorge Velosa

Las expresiones musicales hacen parte de fenómenos culturales tradicionales, donde se manifiestan acontecimientos y hechos de la vida cotidiana y son representados a través de códigos intangibles como el ritmo, la letra, la simbología, considerados dentro de la cultura como los rasgos distintivos espirituales, materiales y afectivos (UNESCO, 2002) que evocan la creatividad humana, portadores de la tradición cultural, los cuales aportan a la construcción de lazos de identidad y son transmitidos a través de prácticas creativas culturales, como un fasto importante que permite a los pueblos y a las personas expresar y compartir con otros sus ideas y valores (UNESCO, 2005)

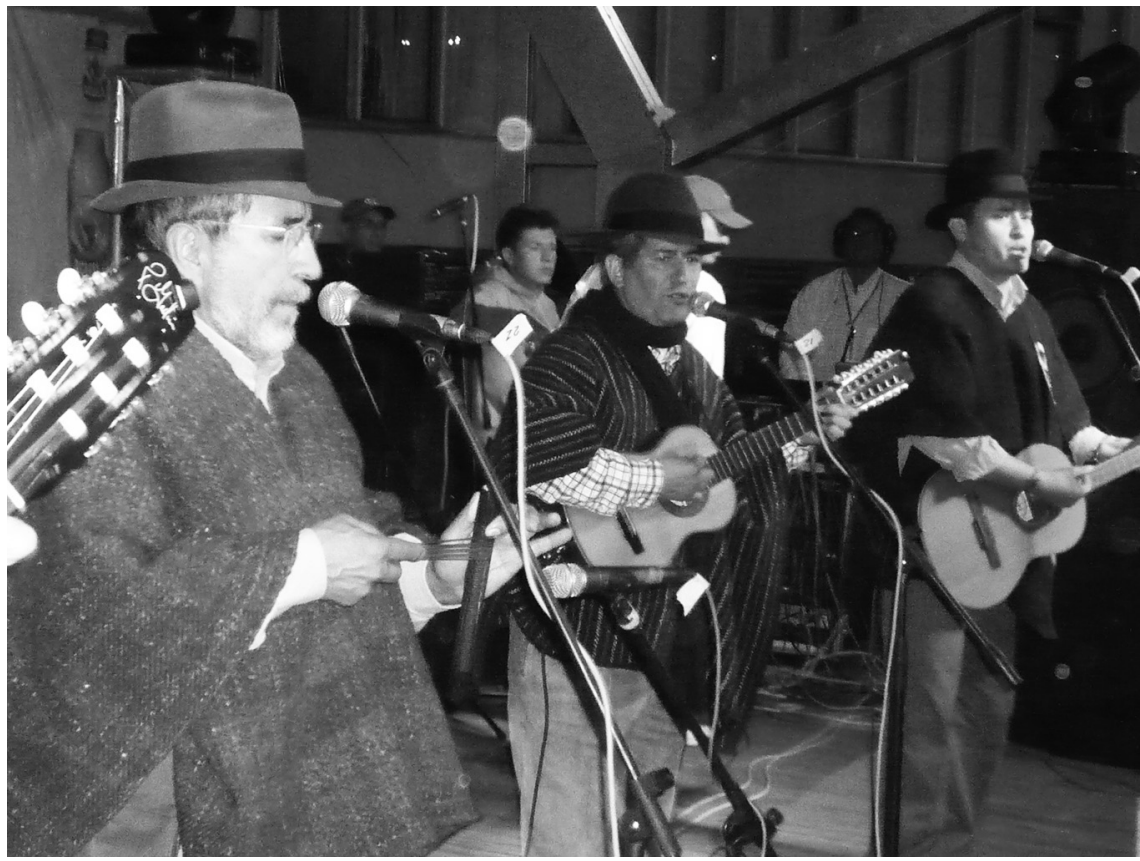

Fuente: Los Carrangueros de Ráquira en las Fiestas del Sol y del Acero en Sogamoso- Boyacá. Julio de 2009 
La carranga como escenario vivo de la tradición e identidad cultural local y regional del departamento

La vinculación de la temática de las prácticas musicales y del folclor, dentro de los temas de investigación, se han fortalecido en las últimas décadas, en la medida en que se ha incorporado a los modos de vida, los sistemas de valores, a los usos sociales y a la oferta del consumo del producto musical, «ya que estimulan al ser humano a pensar en posturas esencialistas que atienden las necesidades, procesos y dinámicas de los cruces de géneros» (San Martín, 1997: 48), los cuales constituyen una gran variedad de manifestaciones, que son percibidas, apropiadas y adoptadas, para alegrar, generar vida, entretener sanamente, evocando un cambio positivo de paz y armonía en la mentalidad de los individuos (Paone, 1999: 13).

La denominación de la música carranguera, se refiere a la Carranga como un regionalismo, que se refiere al animal muerto por enfermedad, accidente, vejez o muerte natural y el cual los dueños para no perderlo completamente y a pesar del riesgo higiénico que esto representaba, lo vendían para hacer embutidos en los muchos sitios de compra de Carranga que existían en la región cundiboyacense, en especial en el municipio de Ubaté, apodado capital mundial de la carranguería como lo afirma Jorge Velosa y se consigna en el estudio realizado por Renato Paone (1999). Sin mbargo, la música popular campesina ha incursionado no solo a nivel local o regional, sino sus canciones han llegado a los máximos escenarios internacionales.

Este género es uno de los más representativos de la cultura cundiboyacence, pues mantiene un fuerte carácter de cohesión e integración social, (González, 2011: 19) el cual emerge normalmente dentro de un ambiente eminentemente colectivo, apoyándose en la construcción de experiencias reales que permiten percibir el alma de una festividad y de hechos existentes que perduran en un legado bajo la tendencia de exteriorizar sentimientos y comportamientos característicos del los individuos, generando una fuerte o débil integración social.

La música carranguera nace de la fusión de la rumba criolla, el bambuco, el torbellino y el merengue campesino del altiplano cundiboyacense y de Santander, liderada por el cantautor, interprete, investigador y folclorólogo Dr. Jorge Velosa Ruiz. Este médico veterinario y su agrupación musical Los Carrangueros de Ráquira - Pueblo de Olleros- reconocidos como los principales exponentes de este género musical, tras haberlo popularizado en una representación o una puesta en escena de lo social, reencarnando las tradiciones campesinas, se convirtió en un legado musical, que sin duda alguna, se considera como un fenómeno de participación masiva donde la integración de ciertos valores dominantes, las contingencias y rupturas sirven para configurar la identidad autóctona y originaria de los pueblos que son la razón de ser de una comunidad (Ocampo López, 2001: 11). 
Este grupo musical ha mostrado con toda pompa la originalidad histórica y cultural del pueblo boyacense, a través de la interpretación armoniosa, estableciendo con el entrecruzamiento de elementos como la letra, la danza, el vestuario, los instrumentos musicales -(guitarra, triple, dulzaina, requinto, guacharaca), las canciones más representativas que enmarcan desde los años setenta, el legado ancestral de los principales pilares de la cultura propia del territorio, encaminadas a garantizar la preservación, protección, promoción, valorización y transmisión del patrimonio cultural inmaterial del territorio (Unesco, 2003).

En honor al maestro Jorge Velosa, en las Ferias de Sogamoso y Festival del Sol y el Acero, que se realiza del 20 al 28 de Julio, nace el Festival de Música Carranguera. A continuación, parte de la canción con las que el autor hace un homenaje a esta tierra y a los campesinos colombianos.

"En mi tierra yo me siento como un rey
un rey pobre pero al fin y al cabo rey
mi castillo es un ranchito de embarrar
y mi reino todo lo que alcanzó a ver.
Por corona tengo la cara de el sol y por capa una ruana sin cardar es mi cetro el cabo de mi azadón es mi trono una piedra de amolar" 3

Según Ocampo (2001), la música popular transita de generación en generación, es autóctona, se originan a partir de acontecimientos, se perpetúa mediante la tradición oral, puesto que permanece como manifestación continua entre el pasado y el presente, se constituye en patrimonio de un pueblo que las canta, baila y las vive, la Unesco define la cultura tradicional y popular "“como el conjunto de creaciones que emanan de una comunidad cultural fundada en la tradición, expresada por un grupo o por individuos y que reconocidamente responden a las expectativas de la comunidad en cuanto a floración de su identidad cultural y social; las normas y los valores se transmiten oralmente, por imitación o de otras maneras, sus formas comprenden, entre otras: la lengua, la literatura, la música, la danza, los juegos, la mitología, los ritos, las costumbres, la artesanía, la arquitectura y otras artes."»(Unesco, 1989). (Recomendación sobre la salvaguardia de la cultura tradicional y popular. París, 15 de noviembre de 1989), ligadas a la música popular, a las costumbres y tradiciones de los pueblos que las emergenven emerger, son figuras frágiles de la cultura popular viviente. Es por ello, que todas estas manifestaciones, ostentan un conjunto de acontecimientos elementos de variados tipos y 
matices donde se congregan diferentes culturas, grupos étnicos y sociales los cuales traen consigo sus tradiciones, creencias y, rituales, formando parte de la memoria que se transmite como herencia de un pueblo.

Velosa, señala que "poco a poco fueron apareciendo más y más seguidores, grupos, concursos, encuentros, programas, investigaciones y así, cantando y decantando, nos fuimos diferenciando de otras tendencias, hasta que se empezó a hablar de la música carranguera, como una figura musical, la cual se convierte en un símbolo emblemático que representa una comunidad, respondiendo a la amalgama de sus costumbres y tradiciones con la identidad cultural".

Parece ser que la Carranguería fue un muy buen negocio a mediados de siglo $\mathrm{XX}$, incluso parece ser más antiguo aun este oficio según escribió Fray Sandalio de las Llagas en sus Crónicas de Boyacá (Época colonial), citado por Obregón (1982). Los que negociaban con Carranga se les llamaba Carrangueros y se les veía como delincuentes por su oficio; tratar con un Carranguero era algo indecoroso por su condición de traficantes de carroña afirma este autor. (Obregón, 1982) en el mismo texto. Desde entonces y por cerca de tres décadas, este estilo musical, ha cobrado importancia, posesionándose y manteniéndose en lugares relevantes de audiencia para los diferentes habitantes de la región y el país.

La armonía de la música carrangera, además de honrar y respetar al campesino, manifiesta la idiosincrasia de éste, ya que en su interpretación jocosa o de doble intención en sus ritmos narra, divierte y propone diferentes ámbitos que ostentan una adhesión profunda por el campo como es el caso de la pirinola; de los amores y quereres como el de Rosita o la Julia; los despechos; además de historias del diario vivir; los contrastes rural-urbano como el de la china que yo tenía se fue pa 'la capital; o tal vez como la perdida de la cucharita; además del cariño por los animales y otras manifestaciones importantes que reflejan hechos militares, como soldado de la patria, así como otros tantos sociales, religiosos y políticos.

Aunque algunas expresiones orales de la humanidad se han adaptado a diversas formas y manifestaciones vivas como la música, la simbología, las representaciones, los usos, las costumbres, los actos festivos y las prácticas sociales, sobreviven como vestigios ante las nuevas culturas, algunas de ellas han logrado transmitirse, adaptándose a nuevas creaciones como la música carranguera, las cuales debido a las transformaciones y paso del tiempo deben ser protegidas, pues cada vez se ven más amenazadas, desvaneciendo la cultura tradicional, es así, que los ritmos propios de la región - música popular- cada vez se va desconociendo y degradando con la subsiguiente pérdida parcial de la identidad cultural. 
En efecto, la música carranguera, encierra un sentido de pertenencia a un grupo social con el cual se comparten rasgos culturales como costumbres, valores y creencias, recreado individual y colectivamente de forma continua. Existen manifestaciones culturales que expresan con mayor intensidad que otras su sentido de identidad, hecho que la diferencia de otras actividades que son parte común de la vida cotidiana. Las representaciones culturales de gran repercusión pública, como la música, la danza, los actos festivos, entre otras, han sido registradas por la Unesco bajo el concepto de Patrimonio Cultural Inmaterial en la convención del 2003, estableciendo como parte de ellos los usos, representaciones, expresiones, conocimientos y técnicas junto con los instrumentos, objetos, artefactos y espacios culturales que les son inherentes, por lo que es importante que las comunidades, los grupos y en los individuos les reconozcan como parte integrante de su patrimonio cultural, infundiéndoles un sentimiento de identidad y continuidad, contribuyendo así a promover el respeto de la diversidad cultural y la creatividad humana (Unesco, 2003: 2).

Las expresiones culturales como las fiestas populares, las cuales propenden por la igualdad, y es la razón fundamental para que se extiendan a toda la sociedad y establezcan para siempre, hacen parte de la cultura tradicional. Las fiestas expresan diferentes códigos y formas de identidad de un grupo, por lo tanto se debe tomar medidas para salvaguardar, conservar, proteger y respetar no solo por parte de quienes interpretan y reafirman el carácter plural de su propia identidad, sino de las entidades con las cuales se mantienen vínculos de entendimiento y afecto. De esta manera se fomenta la diversidad cultural y se garantiza el patrimonio viviente.

En este sentido, la música es considerada parte del Patrimonio Inmaterial de una localidad, pues ella hace parte de manifestaciones y testimonios, plasmados en composiciones y melodías que desbordan ideas y prácticas que resaltan fuertemente sentimientos, emociones y efusividad propios de cada ser, acompañadas de espacios y elementos culturales inherentes a la cotidianidad, las cuales se dan a conocer dependiendo de un motivo o hechos, transformándose en una riqueza invaluable para la identidad cultural de una comunidad.

Actualmente existen numerosos grupos musicales de carranga, este género se ha convertido no solo como referencia para otras tendencias musicales, sino como apoyo de investigaciones académicas (Sánchez, 2008: 111), también como una alternativa para mantener viva la tradición y de una u otra manera contribuir con la identidad cultural como parte esencial del patrimonio inmaterial local, regional y nacional.

En conclusión, la música popular como la carranga, hace parte de un linaje cultural de profundo significado ritual para las comunidades o grupos, estas expresiones contienen un fuerte compromiso social, las cuales no solo 


\section{Francy Marizol Rojas Parra}

La carranga como escenario vivo de la tradición e identidad cultural local y regional del departamento de Boyacá. Artículo de reflexión.

infunden un sentimiento de identidad y continuidad sino que además son un auténtico crisol de la diversidad cultural, inherentes al patrimonio vivo el cual evoluciona constantemente y, al mismo tiempo, se ve amenazado por las repercusiones de la globalización, así como de los diversos conflictos que convocan a su disgregación cultural.

\section{Referencias bibliográficas}

González, M. (Coord.). 2011. Fiestas y nación en América Latina: las complejidades en algunos ceremoniales de Brasil, Bolivia, Colombia, México y Venezuela. Bogotá: Intercultura.

Martín-Barbero, J. 1998. Cultura, sociedad y medios. Bogotá: Universidad Nacional de Colombia.

Obregón, E. 1982, junio 12. La rumba carranguera, EL Mundo Semanal .

Ocampo, J. 2001. El imaginario en Boyacá: la identidad del pueblo boyacense y su proyección en la simbología regional. Vol. I y II. Bogotá: Universidad Distrital «Francisco José de Caldas».

Paone, R. 1999. La música carranguera. Monografia de grado, programa de Música. Medellín: Escuela Popular de Arte.

Rojas, F. La fiesta: valoración de su impacto histórico, cultural y social. Memorias festivas de Sogamoso - Boyacá- 1960-2012.

San Martín, J.E. 1997. Psicosociología del ocio y el turismo. Málaga: Ediciones

Sánchez, T. y Acosta, A. 2008. Música popular campesina. Usos sociales, incursión en escenarios escolares y apropiación por los niños y niñas. La propuesta musical de Velosa y Los Carrangueros. Revista Latinoamericana de Ciencias Sociales, Niñez y Juventud, Vol 6 (1): 111-146.

Unesco. 2005. Convención sobre la protección y promoción de la diversidad de las expresiones culturales. París, 20 de octubre. http://unesdoc.unesco.org/ images/0014/001429/142919s.pdf

Unesco. 2003. Convención para la Salvaguardia del Patrimonio Cultural Inmaterial. París 17 de octubre. http://www.unesco.org/new/es/santiago/culture/intangibleheritage/convention-intangible-cultural-heritage/

Unesco. 2002. Declaración Universal de la Unesco sobre la Diversidad Cultural. 4 de Septiembre. http://portal.unesco.org/es/ev.php-URL_ID=13179\&URL_DO=DO_ TOPIC\&URL_SECTION=201.html 
Unesco. 1998. Conferencia Intergubernamental sobre Politicas Culturales para el Desarrollo. Estocolmo del 30 de marzo. http://unesdoc.unesco.org/ images/0011/001139/113935So.pdf

Unesco. 1995. Nuestra Diversidad Creativa. Comisión Mundial de Cultura y Desarrollo. http://unesdoc.unesco.org/images/0010/001055/105586sb.pdf

Unesco. 1989. Recomendación sobre la salvaguardia de la cultura tradicional y popular. 15 de noviembre. http://portal.unesco.org/es/ev.php-URL_ID=13141\&URL_DO=DO_ TOPIC\&URL_SECTION=201.html

Unesco. 1982. Declaración de México sobre las políticas culturales. Conferencia mundial sobre las políticas culturales (de julio-6 de agosto). http://portal.unesco.org/ culture/es/files/35197/11919413801mexico_sp.pdf/mexico_sp.pdf 